\title{
Nutrient Status and perceptions of energy and macronutrient intake in a Group of Collegiate Female Lacrosse Athletes
}

\author{
Andrew R. Jagim ${ }^{1 *}$ (D), Hannah Zabriskie², Brad Currier², Patrick S. Harty ${ }^{2}$, Richard Stecker ${ }^{2}$ and Chad M. Kerksick ${ }^{2}$
}

\begin{abstract}
Background: The purpose of this study was to compare nutritional intakes against recommended values as well as between the perceived intake and needs of female lacrosse players.

Methods: Twenty female NCAA Division II lacrosse players (20.0 $\pm 1.7 \mathrm{yrs} ., 169.7 \pm 6.4 \mathrm{~cm} ; 69.9 \pm 10.7 \mathrm{~kg} ; 27.5 \pm 3.3 \%$ fat) completed a four-day monitoring period during in-season. Athletes were outfitted with an activity monitor over four consecutive days and completed four-day food records to assess total daily energy expenditure (TDEE) and dietary intake. Body composition was assessed and used to calculate recommended dietary intakes. Actual intake was self-reported using a commercially available food tracking program (MyFitnessPal@, USA). Daily average values were calculated for total and relative energy, protein, carbohydrate, and fat intake. These values were then compared to published nutritional recommendations established by the International Society of Sports Nutrition. Appropriate pairwise comparisons were made depending on the normality of the distribution.

Results: Athletes ate significantly less than recommended values for energy, carbohydrates and protein. $(p<0.001)$. Significant discrepancies $(p<0.001)$ were also observed between perceptions of intake versus actual intake.

Conclusions: Athletes significantly underestimated perceived intake of dietary fat and carbohydrate when compared to perceived needs. Massive standard deviations and ranges were observed, suggesting that some athletes lack a basic understanding of their daily needs. Results from this data suggest that collegiate athletes lack appropriate understanding of basic nutrition needs and could benefit from basic nutrition education as it pertains to their health and performance.
\end{abstract}

Keywords: Nutrition, Calories, Energy, Macronutrients, Females, Athletes, Energy balance, Energy expenditure, Energy availability

\section{Background}

Dietary practices have a profound impact on athlete health and performance $[1,2]$. Due to increased physical demands, athletes achieve daily energy expenditures that require above-average energy and macronutrient intakes to sustain training, enhance recovery, and maintain performance [1]. To address these specialized dietary requirements, professional organizations such as the International Society of Sports Nutrition (ISSN) and the International Olympic Committee (IOC) have established

\footnotetext{
* Correspondence: jagim.andrew@mayo.edu

${ }^{1}$ Sports Medicine, Mayo Clinic Health System, 109 Theater Road, Onalaska, WI 54650, USA

Full list of author information is available at the end of the article
}

dietary recommendations for athletes [1-3]. However, many athletes struggle to follow dietary recommendations, and several studies have shown that actual nutrient intakes of collegiate athletes and reported energy and macronutrient intake levels fall below the recommended daily allowance (RDA) or recommendations made by professional organizations [4-8]. These findings should be interpreted in light of the fact that athletes may have higher energy, macronutrient, and micronutrient requirements than RDA-derived values, and therefore, their nutrient intakes may need to be even higher than RDA values [1]. Previous research has indicated that female athletes are at particular risk for nutritional deficiencies resulting from a high volume of training coupled with

(C) The Author(s). 2019 Open Access This article is distributed under the terms of the Creative Commons Attribution 4.0 International License (http://creativecommons.org/licenses/by/4.0/), which permits unrestricted use, distribution, and 
inadequate energy and macronutrient intake $[9,10]$. Such deficiencies can compromise performance, impair recovery, negatively influence endocrine function, and increase susceptibility to injuries and illness [10].

Factors such as inadequate nutrition knowledge, logistical challenges (i.e. traveling, time spent practicing, accessibility etc.), physique requirements, social pressures, and inadequate financial resources are often listed as key barriers to acceptable nutrition within elite athletes [11, 12]. Furthermore, previous $[5,12-16]$ reports indicate that athletes struggle to correctly identify recommended levels of macronutrient intakes for their sport or correctly answer questions about basic nutrition knowledge, supplements, weight management, and hydration [13, 16]. A failure to understand how dietary requirements fluctuate with the changing demands of training and competition may result in situations where athletes are chronically under-fueled. Unfortunately, even wellinformed athletes may not translate nutrition knowledge to a sufficient dietary intake due to the barriers outlined previously. Moreover, an athlete's perceptions of their diet may not align with their nutritional requirements or their actual intake. These issues are likely further magnified at the collegiate level where nutrition-based support services or educational resources are often not available to student athletes.

Currently, there is limited research evaluating how effectively female team sport athletes meet sport-specific nutritional recommendations. Furthermore, there is a paucity of research exploring the accuracy of athletes' perception of their energy and macronutrient needs, as well as their perceived dietary intake compared to their actual consumption. Therefore, the purpose of this study was two-fold: 1) To compare calculated ISSN recommendations for daily energy and macronutrient intake to actual in-season dietary intake of female lacrosse players; 2) To identify the discrepancies that exist in female collegiate athletes between their perceived energy and macronutrient needs as well as between their perceived and actual intake of energy and macronutrients.

\section{Methods}

\section{Experimental design}

All athletes were outfitted with an activity monitor (Acti-Heart, CamNTech, Inc.) during in-season competition and team activities over four consecutive days (worn continuously). The monitoring period consisting of 2 weekdays and 2 weekend days during May of the 2018 season and was used to assess total daily energy expenditure (TDEE). Participants were assessed for body composition, which was used to calculate recommended intake values $[1,2]$. Participants also recorded dietary intake (food and fluid) during the four-day monitoring period using a commercially available food tracking program. Daily average values were calculated for total and relative energy, protein, carbohydrate, and fat intake. These values were then compared to nutritional recommendations provided in position stands and summary statements put forth by the ISSN [2] and American College of Sports Medicine (ACSM) [1]. To avoid bias, we identified energy intake recommendations for low, middle, and high levels of activity (40,50, and $60 \mathrm{kcals} / \mathrm{kg} /$ day, respectively). The same methods were used to determine intakes for carbohydrate $(4,6$, and $8 \mathrm{~g} / \mathrm{kg} /$ day $)$, protein $(1.4,1.6$, and $1.8 \mathrm{~g} / \mathrm{kg} /$ day $)$, and fat $(15,25$, and $35 \%$ calories), respectively. Recommendations for energy intake were compared to measured TDEE from activity monitors. In addition, each athlete recorded their perceived nutrient needs and their perceived nutrient intake over the collection period by completing a questionnaire.

\section{Study participants}

Twenty female NCAA Division II Lacrosse players $(20.0 \pm 1.7$ years, $\quad 169.7 \pm 6.4 \mathrm{~cm} ; \quad 69.9 \pm 10.7 \mathrm{~kg} ; \quad 27.5 \pm$ $3.3 \%$ body fat) completed all aspects of testing. All athletes were medically cleared and participated in all team activities during the study period. Team members who were not able to participate in all team activities were excluded from the study. Prior to testing, all athletes provided written consent and the study protocol was approved by the Lindenwood University Institutional Review Board.

\section{Dietary intake}

Dietary energy intake was assessed from four-day diet logs completed during the same days that energy expenditure was monitored. Subjects were given food log packets that illustrated how to accurately record portion sizes of various foods and beverages consumed. Athletes logged all calorie-containing food and beverages for four consecutive days using the MyFitnessPal smartphone application (MyFitnessPal@, USA). Four-day averages were computed for energy, carbohydrate, protein, and fat intake and both raw values and values normalized to body mass in kilograms were used in the analysis.

\section{Resting energy expenditure}

All resting energy expenditure (REE) measures were completed using a ParvoMedics TrueOne 2400 metabolic measurement system (Sandy, UT). Each morning the indirect calorimetry system was calibrated to ensure that variations in measured oxygen and carbon dioxide and the flow rate were less than $2 \%$ different than the previous calibration. A clear plastic hood and drape was placed over each participant's head and shoulders with the flow rate on the dilution pump set to maintain approximately $0.8-1.2 \%$ carbon dioxide in the expired gases. Study participants remained awake and motionless 
in a supine position for $20-25$ min while data were collected at 1-min intervals. The recorded data were visually inspected to identify a 5-min window during the final 10 minutes of data collection where $\mathrm{VO}_{2}$ (in $\mathrm{L} /$ min) changed less than $5 \%$ to identify a daily average of REE (in $\mathrm{kcal} /$ day). Participants were instructed to fast from all energy-containing foods and fluids for a minimum of 8 hours prior to the test and did not exercise or perform physical activity for $24 \mathrm{~h}$ prior to the test. All REE assessments occurred within 2 weeks of activity energy expenditure assessment and self-reporting of dietary intake.

\section{Body composition (DEXA)}

Body composition assessments were completed using dual-energy x-ray absorptiometry (DEXA). To standardize testing conditions, participants observed an 8-h fast of energy-containing food and fluid and avoided exercise for at least 24 h [17] prior to assessments. All DEXA scans occurred within 2 weeks before or after activity energy expenditure assessment and self-reporting of dietary intake. Calibration procedures were completed each day before testing, and all DEXA scans were completed using the Discovery DEXA System (HOLOGIC, Inc., Bedford, MA) and analyzed using its accompanying software (Hologic APEX Software, Version 4.5.3, HOLOGIC, Bedford, MA) to determine whole-body levels of bone, fat, and fat-free masses along with body fat percentages. Previous testretest reliability analysis of these procedures yielded intraclass correlation coefficients of $\geq 0.998$.

\section{Activity energy expenditure}

In-season activity energy expenditure was assessed during the same four-day period used for dietary intake assessment, consisting of two weekdays and two weekend days. Whenever possible, the assessment period contained a game day, an off day, and two practice days using only accelerometer data from the physical activity monitors (Acti-Heart, CamNTech, Inc., Boerne, TX). Each monitor was worn on the left side of the athlete's chest below their left breast. The monitors were attached at the level of the xiphoid at the anterior midline and laterally positioned at the anterior axillary line using standard ECG electrodes. Both electrode locations were adjusted to ensure the lead wire was parallel to the ground. The Acti-Heart software computes activity energy expenditure, predicts resting metabolic rate using the equation of Schofield [18], and assigns a value for thermic effect of food that is fixed at $10 \%$ of the computed TDEE. TDEE and activity energy expenditure (AEE) values were used to create an average of TDEE and AEE over the entire 4-day period. Previously published work by Assah et al. $[19,20]$ in a large group of free-living adults revealed that the monitors were valid in comparison to doubly labeled water.

\section{Perceptions of dietary intake and dietary needs}

A brief questionnaire was developed to compare the athletes' perceptions of their nutrient needs to ISSN derived energy and macronutrient intake recommendations based on the requirements of their sport. A secondary aim of the questionnaire was to assess each athlete's perceived energy and macronutrient intake on a typical day and then compared to the established dietary guidelines for their sport.

\section{Statistical analysis}

From the original 22 participants recruited to complete this study, two participants were removed from the analysis due to noncompliance with the testing protocol. Three additional participants did not complete perceived nutrition intake questionnaires. Thus, a sample size of 17 was used for all calculations involving perceived intake, while a sample size of 20 was used for all other calculations. To aid in understanding of the magnitude of discrepancy with perceived needs and intake, only raw values were used for computations (i.e., kcals/day and grams/day). A $p$-value of $<0.05$ was used to determine statistical significance. SPSS V.25 for Windows (Armonk, NY) and Microsoft Excel (Seattle, WA) were used to complete all statistical analyses. All normally distributed data are presented as means \pm standard deviations and all non-normally distributed data are presented as median \pm interquartile range (IQR). The Shapiro-Wilk test was used to determine normality. When normality was confirmed, paired samples t-tests were used to assess differences between groups. When the normality assumption was violated, Wilcoxon Signed Rank tests were used to assess differences between the non-normally distributed variables.

\section{Results}

Significant differences $(p<0.001)$ were observed between all energy and macronutrient recommendations when compared to actual intakes. These differences were present for both total and relative daily values. For energy and all macronutrient recommendations, athletes consumed well below the recommendations (Table 1). Absolute TDEE in this study was determined to be $2582 \pm 303 \mathrm{kcals} /$ day, (95\% CI: 2441, 2724) while relative energy expenditure was $37.9 \pm 4.7 \mathrm{kcal} / \mathrm{kg} /$ day $(95 \% \mathrm{CI}$ : $35.7,40.1)$. The ISSN has provided general energy intake recommendations of 40,50 , and $60 \mathrm{kcals} / \mathrm{kg} /$ day for athletes participating in low, medium, and high levels of training volume, respectively. Using the measured body mass levels of athletes in our cohort, these recommended energy intake values translated into $2756 \pm 403$ kcals/day (95\% CI: 2567, 2945), $3445 \pm 504 \mathrm{kcals} /$ day (95\% CI: 3209, 3681), and $4134 \pm 605$ kcals/day (95\% CI: 
Table 1 Comparison of recommended dietary intake versus actual intake $(n=20)$

\begin{tabular}{|c|c|c|c|c|c|}
\hline \multirow[b]{2}{*}{ Total Energy Intake (kcal/d) } & \multirow{2}{*}{$\begin{array}{l}\text { Actual Intake* } \\
2161 \pm 392(1978,2344)\end{array}$} & \multicolumn{2}{|c|}{ Recommended§ } & \multirow{2}{*}{$\begin{array}{l}\text { Delta Intake (Actual - Recommended) } \\
-595 \pm 605(-878,-312)\end{array}$} & \multirow{2}{*}{$\frac{p \text { value }}{<0.001}$} \\
\hline & & Low & $2756 \pm 403(2567,2945)$ & & \\
\hline & & Moderate & $3445 \pm 504(3209,3681)$ & $-1284 \pm 685(-1604,-963)$ & $<0.001$ \\
\hline & & High & $4134 \pm 605(3851,4417)$ & $-1973 \pm 771(-2333,-1612)$ & $<0.001$ \\
\hline \multirow[t]{3}{*}{ Relative Energy Intake $(\mathrm{kcal} / \mathrm{kg} / \mathrm{d})$} & $32.1 \pm 7.9(28.4,35.6)$ & Low & 40 & & \\
\hline & & Moderate & 50 & & \\
\hline & & High & 60 & & \\
\hline \multirow[t]{3}{*}{ Total CHO Intake (g/d) } & $236 \pm 74(201,270)$ & Low & $275.6 \pm 40.3(257,294)$ & $-40.0 \pm 83.4(-79,-0.94)$ & 0.05 \\
\hline & & Moderate & $413.4 \pm 60.5(385,442)$ & $-178 \pm 94(-222,-134)$ & $<0.001$ \\
\hline & & High & $551.2 \pm 80.7(514,589)$ & $-316 \pm 108(-366,-265)$ & $<0.001$ \\
\hline \multirow[t]{3}{*}{ Relative $\mathrm{CHO}$ Intake $(\mathrm{g} / \mathrm{kg} / \mathrm{d})$} & $3.48 \pm 1.19(2.92,4.03)$ & Low & 4.0 & & \\
\hline & & Moderate & 6.0 & & \\
\hline & & High & 8.0 & & \\
\hline \multirow[t]{3}{*}{ Total PRO Intake (g/d) } & $78.8 \pm 19.6(69.6,88.0)$ & Low & $96.5 \pm 14.1(90,103)$ & $-17.7 \pm 28.2(-31.9,-4.5)$ & 0.011 \\
\hline & & Moderate & $110.2 \pm 16.1(103,118)$ & $-31.4 \pm 29.8(-45.4,-17.5)$ & $<0.001$ \\
\hline & & High & $124.0 \pm 18.1(116,133)$ & $-45.2 \pm 31.4(-59.9,-30.5)$ & $<0.001$ \\
\hline \multirow[t]{3}{*}{ Relative PRO Intake (g/kg/d) } & $1.18 \pm 0.38(1.00,1.36)$ & Low & 1.4 & & \\
\hline & & Moderate & 1.6 & & \\
\hline & & High & 1.8 & & \\
\hline \multirow[t]{3}{*}{ Total Fat Intake (g/d) } & $87.9 \pm 22.8(77.3,98.6)$ & Low & $36.0 \pm 6.5(33.0,39.1)$ & $51.9 \pm 19.7(42.7,61.1)$ & $<0.001$ \\
\hline & & Moderate & $60.0 \pm 10.9(54.9,65.1)$ & $27.9 \pm 18.7(19.1,36.6)$ & $<0.001$ \\
\hline & & High & $84.1 \pm 15.2(76.9,91.2)$ & $3.9 \pm 18.6(-4.9,12.6)$ & 0.37 \\
\hline \multirow[t]{3}{*}{ Relative Fat Intake (g/kg/d) } & $1.31 \pm 0.41(1.11,1.50)$ & Low & $15 \%$ & & \\
\hline & & Moderate & $25 \%$ & & \\
\hline & & High & $35 \%$ & & \\
\hline
\end{tabular}

§Recommend values are derived through a combination of published review articles $[1,2]$ and clinical experience. Delta Intake $=$ Actual intake - Recommended intake. All variables exhibited normal distributions using Shapiro-Wilk tests $(p>0.05)$. Data presented as mean \pm SD with the $95 \%$ confidence interval presented in parentheses below the mean \pm SD. ${ }^{*}=$ As outlined in statistical analysis section, dietary intake values reported in Table 1 and Table 2 are expected to be slightly different due to the removal of three participants who failed to complete all of the required perceived nutrition assessment

3851, 4417), respectively for the low, medium, and high energy intake recommendations. When compared to self-reported dietary intake ( $2161 \pm 392 \mathrm{kcals} /$ day $)$, the athletes in the current study reported the intake of less energy than all recommended levels. When compared to the lowest recommended intake, the mean difference between this recommended intake amount and the selfreported intake amount was $-595 \pm 605 \mathrm{kcals} /$ day $(95 \%$ CI: $-878,-312 \mathrm{kcals} /$ day). The mean differences between the medium and high recommended energy intake levels and the self-reported energy intake level were $-1284 \pm 685 \mathrm{kcals} /$ day $(-1604,-963 \mathrm{kcals} /$ day $)$ and $-1973 \pm 771 \mathrm{kcals} /$ day $(-2333,-1612 \mathrm{kcals} /$ day $)$, respectively. All recommended energy intake levels were significantly different than the reported energy intake levels $(p<0.001)$.

In addition, discrepancies were found between the actual reported carbohydrate intake and recommended intake. Paired samples t-tests between the reported carbohydrate intake (236 \pm 73 g/day, 95\% CI: 201, 270 g/ day) and the low, moderate and high recommended amounts were all statistically significant (Table 1). Similarly, paired samples t-tests between the reported protein intake ( $79 \pm 20 \mathrm{~g} /$ day, $95 \% \mathrm{CI}: 70,88 \mathrm{~g} /$ day) and the low, moderate and high recommended amounts were all statistically significant (Table 1). Finally, paired samples ttests between the reported fat intake $(88 \pm 23 \mathrm{~g} /$ day, $95 \%$ CI: $77,99 \mathrm{~g} /$ day) and the low and moderate recommended amounts were found to be statistically significant, while no significant difference was found between actual fat intake and the high recommended amount $(p=0.37)$.

Because the perceived needs and intake data were not normally distributed, we used Wilcoxon Signed Rank tests were used to assess differences between perceived needs and perceived intake as well as between perceived intake and actual intake. As seen in Table 2, widespread variability existed within the perceived needs and intake data resulting in large deviations from normality. Significant differences were observed between the perceived 
Table 2 A comparison of perceived versus actual dietary intake $(n=17)$

\begin{tabular}{|c|c|c|c|c|c|}
\hline & Perceived Needs & Perceived Intake & $\begin{array}{l}\text { Delta Perceived } \\
p \text {-value }\end{array}$ & Actual Intake & $\begin{array}{l}\text { Delta Intake } \\
p \text {-value }\end{array}$ \\
\hline Energy Intake (kcals/day) & $2000 \pm 300$ & $\begin{array}{l}2214 \pm 679^{a} \\
(1,866,2564)\end{array}$ & $\begin{array}{l}-30.8 \pm 563^{a} \neq \\
(-320,259) 0.42\end{array}$ & $\begin{array}{l}2137 \pm 418^{a} \\
(1,922,2351)\end{array}$ & $\begin{array}{l}78.0 \pm 693^{a} \neq \\
(-278,434) 0.65\end{array}$ \\
\hline Carbohydrate Intake (grams/day) & $80 \pm 213$ & $150 \pm 280$ & $-129 \pm 228^{*} 0.004$ & $\begin{array}{l}242 \pm 73^{a} \\
(204,279)\end{array}$ & $-30 \pm 240 \neq 0.33$ \\
\hline Protein Intake (grams/day) & $45 \pm 41$ & $30 \pm 32.5$ & $-33.8 \pm 42.3^{*} 0.39$ & $\begin{array}{l}77.7 \pm 20.4^{a} \\
(67,88)\end{array}$ & $-42.8 \pm 42.3 \neq 0.98$ \\
\hline Fat Intake (grams/day) & $30 \pm 40$ & $50 \pm 63$ & $\begin{array}{l}22.9 \pm 120^{*} \\
(-84.6,38.8) 0.007\end{array}$ & $\begin{array}{l}86.1 \pm 22.4^{a} \\
(75,98)\end{array}$ & $\begin{array}{l}62.1 \pm 306 \neq \\
(-95,219) 0.42\end{array}$ \\
\hline
\end{tabular}

$\mathrm{a}=$ Data is normally distributed and presented as means \pm standard deviation with the $95 \%$ confidence interval in parentheses. Delta perceived $=$ perceived needs - perceived intake. Delta intake $=$ perceived intake - actual intake. ${ }^{*}=p$-value from Wilcoxon Signed Rank Test (non-normally distributed variables). $\neq=p$-value from paired samples t-test (normally distributed data). When one value was non-normally distributed and another variable was normally distributed, a paired samples t-test was used to assess differences between the two means

needs and perceived intakes for absolute carbohydrate $(p=0.004)$ and absolute fat $(p=0.007)$. No significant differences were observed between perceived intake and actual intake for energy, carbohydrate, protein, or fat intake. All perceived needs, perceived intake and actual dietary intake data are provided in Table 2.

\section{Discussion}

The primary aim of the current study was to evaluate the ability of collegiate female lacrosse players to meet nutritional recommendations established by the ISSN. Secondary aims were to investigate the accuracy of the athletes' perception of their nutritional requirements and to compare their perceived intake to their actual dietary intake. This investigation found that the athletes failed to meet energy and macronutrient recommendations put forth by professional organizations. However, significant deviations were observed when estimated energy requirements were based off the "moderate" or "high" activity levels and compared to the measured TDEE. In this respect, the predicted daily energy requirement that corresponded with the low activity level (40 kcal $/ \mathrm{kg}$ of bodyweight) was similar to the mean (SD) measured TDEE of $37.9 \pm 4.7 \mathrm{kcals} / \mathrm{kg}$ of bodyweight. This level of total daily energy expenditure appears to remain fairly consistent, even throughout the duration of a season in female lacrosse players [21] with average game energy expenditures ranging between 800 and $1000 \mathrm{kcals} /$ game. Even when using a conservative "low" activity level to estimate energy requirements, the athletes in the current study still failed to meet energy requirements, as their mean energy intake was $32.1 \pm 7.9$ $\mathrm{kcal} / \mathrm{kg}$. Previous work has also reported an inability of athletes to meet nutritional guidelines for their sport [4, $8,22-25]$, as consuming adequate energy and carbohydrates appears to be a challenge for many athletes [26]. Beals and colleagues [24] noted that female adolescent volleyball players largely failed to meet recommended intakes for energy, carbohydrates and protein and presented with several micronutrient deficiencies. Similarly, Clark et al. [7] determined that many female collegiate soccer players also failed to meet carbohydrate recommendations, but did meet the Dietary Reference Intake for energy intake for individuals with an "active lifestyle" (37 $\mathrm{kcal} / \mathrm{kg} /$ day). This is in alignment with the results of the current study, as this recommended value is near the ISSN recommendation of $40 \mathrm{kcal} / \mathrm{kg} /$ day that best aligned with the TDEE exhibited by the current players during training $(37.9 \pm 4.7 \mathrm{kcal} / \mathrm{kg} /$ day). Valliant et al. [23] also reported that a group of female collegiate volleyball players failed to meet dietary requirements for energy, carbohydrate, and protein.

It is not well understood whether such nutritional issues are a result of a lack of knowledge, a misunderstanding of dietary behaviors, or the result of other negative influences and bad habits. In the current study, widespread discrepancies were found between athletes' perceptions of their energy and macronutrient needs and their actual dietary intakes as well as between their perceived nutrient intakes and actual intakes. In particular, the athletes' perceived daily carbohydrate and fat needs and perceived dietary intakes displayed massive standard deviations and robust ranges, suggesting that some athletes lack even the most basic understanding of their daily nutritional needs. Moreover, the athletes in the current study perceived their protein needs to be less than what they were consuming. Disparities were also present between an athlete's perceptions of their macronutrient needs when compared to recommended intakes as well as between their perceived nutrient intake and their actual intake. However, the athletes did seem to possess a better grasp of their energy needs, as no differences were found between their perceived energy intake, perceived energy need, and actual energy intake.

Results from this study suggest that collegiate athletes lack appropriate understanding of basic nutrition needs and could likely benefit from basic nutrition education. Targeted sport nutrition education interventions have 
been shown to exert a positive impact on the nutritional knowledge and dietary intake of collegiate athletes [23, $27,28]$. For example, Abood et al. [28] observed that an 8 -week ( $1 \mathrm{~h}$. per week) nutrition education program improved nutrition knowledge and resulted in subsequent positive dietary changes in NCAA Division I female soccer players, though deficiencies in total energy and macronutrient intake were still observed. Similarly, Valliant et al. [23] reported a significant improvement in sports nutrition knowledge as well as total energy and macronutrient intake in NCAA Division I female Volleyball players following an off-season nutrition intervention program. Most recently, Rossi et al. [27] observed significant improvements in nutritional intake, body composition, and performance following a single 90-min sport nutrition education intervention in NCAA Division I baseball players. However, an athlete's nutrition knowledge may only slightly influence dietary intake, as logistical reasons such as time, cooking skills, and financial constraints could prevent a well-informed athlete from eating effectively $[11,12]$.

It is tempting to simply recommend that all universities employ a full-time registered dietician or sports nutritionist, as access to these professionals can improve nutrition knowledge [29, 30]. For many athletic departments, however, these positions are not fiscally possible. Consequently, athletes at these schools tend to rely upon their sport coaches and/or strength and conditioning professionals who may not have enough knowledge to provide appropriate guidance [16]. Even with the available nutritional support staff or educational resources, adherence to nutritional guidelines may still be an issue, as many collegiate athletes are in a unique transitional stage in their lives where they may be tasked with purchasing, preparing, and making decisions about food for the first time. Thus, it is worth noting that even if the athletes have an appropriate level of understanding of nutrition, they may still opt for lower quality options or fail to realize they are not meeting their perceived dietary needs as previously observed and supported by the results of the current study [12].

The use of self-reported dietary intakes is a limitation of the current study as previous research has indicated that dietary logs may be susceptible to underreporting in athlete populations [31]. Additionally, little information is currently available regarding the physiological demands of women's lacrosse. As a result, there are not sport-specific nutritional recommendations available for women's lacrosse which is also a limitation of the current study. Comparisons are often made between soccer and lacrosse as they are both intermittent teambased field sports consisting of short bursts of highintensity sprinting intermixed with jogging [32]. Female lacrosse players also exhibit anthropometric and fitness characteristics that are similar to women's basketball [32].Therefore the nutritional recommendations used in the current study should reflect the nutritional requirements of collegiate female lacrosse players as the ISSN recommendations are designed to meet the needs of an athlete training at this level $[2,33]$. Furthermore, we provided ranges of nutritional recommendations to avoid any bias and present multiple tiers of nutritional requirements based on changes in training level. Future research should focus on examining the physiological demands and sport-specific nutritional requirements of team sports, especially in female athletes.

\section{Conclusions}

The results of this study clearly indicate that many collegiate athletes may lack appropriate understanding of basic nutrition needs and could benefit from basic nutrition education, which would likely reduce training related injuries and optimize performance. Because proper sport nutrition is a vital to ensure peak performance and recovery, qualified professionals face the challenge to educate athletes regarding the benefits of a nutrientdense diet that meets appropriate caloric needs. As smaller institutions frequently do not have the resources to hire full-time nutritional staff, it may be beneficial for coaches to offer a nutrition-education program to ensure their players are meeting the energy requirements for their body size and level of training.

\section{Abbreviations}

AEE: activity energy expenditure; DEXA: dual-energy $\mathrm{x}$-ray absorptiometry; ISSN: International Society of Sports Nutrition; NCAA: National Collegiate Athletic Association; RDA: recommended daily allowance; REE: resting energy expenditure; TDEE: total daily energy expenditure

\section{Acknowledgements}

We would like to acknowledge the women's lacrosse team at Lindenwood University for their willingness to participate in this study.

\section{Authors' contributions}

ARJ was involved with experimental design, data collection, data analysis and manuscript preparation. $\mathrm{HZ}$ was responsible for data collection, data analysis and manuscript preparation. BC assisted with data collection. PSH assisted with manuscript preparation. RS assisted with data collection. CMK assisted with data analysis and manuscript preparation. All authors read and approved the final manuscript.

\section{Authors' information}

ARJ is the Director of Sports Medicine Research at Mayo Clinic Health System in Onalaksa, Wisconsin. HAZ and PSH are graduate students in the Human Performance program at Lindenwood University. BC is an undergraduate student in the Exercise Science program at Lindenwood University. RS is the Exercise and Performance Nutrition Laboratory Coordinator at Lindenwood University. CMK is an Associate Professor of Exercise Science and Director of the Human Performance program and Exercise and Performance Nutrition Laboratory at Lindenwood University.

Funding

No funding was received for this study. 


\section{Availability of data and materials}

The datasets used and/or analyzed during the current study are available from the corresponding author on reasonable request.

\section{Ethics approval and consent to participate}

This study was approved by the Institutional Review Board at Lindenwood University and all participants provided written consent prior to their participation in the study.

\section{Consent for publication}

Not applicable

\section{Competing interests}

The authors declare they have no competing interests.

\section{Author details}

'Sports Medicine, Mayo Clinic Health System, 109 Theater Road, Onalaska, WI 54650, USA. ${ }^{2}$ Exercise and Performance Nutrition Laboratory, Lindenwood University, St. Charles, MO, USA.

Received: 15 April 2019 Accepted: 26 September 2019

Published online: 15 October 2019

\section{References}

1. Thomas DT, Erdman KA, Burke LM. American College of Sports Medicine joint position statement. Nutrition and athletic performance. Med Sci Sports Exerc. 2016;48(3):543-68.

2. Kerksick CM, et al. ISSN exercise \& sports nutrition review update: research \& recommendations. J Int Soc Sports Nutr. 2018;15(1):38.

3. Potgieter S. Sport nutrition: a review of the latest guidelines for exercise and sport nutrition from the American College of Sport Nutrition, the International Olympic Committee and the International Society for Sports Nutrition. S Afr J Clin Nutr. 2013;26(1):6-16.

4. Hickson JF Jr, Schrader J, Trischler LC. Dietary intakes of female basketball and gymnastics athletes. J Am Diet Assoc. 1986;86(2):251-3.

5. Cole CR, et al. Evaluation of dietary practices of National Collegiate Athletic Association Division I football players. J Strength Cond Res. 2005;19(3):490-4.

6. Nowak RK, Knudsen KS, Schulz LO. Body composition and nutrient intakes of college men and women basketball players. J Am Diet Assoc. 1988;88(5): 575-8.

7. Clark M, et al. Pre- and post-season dietary intake, body composition, and performance indices of NCAA division I female soccer players. Int I Sport Nutr Exerc Metab. 2003:13(3):303-19.

8. Jagim AR, Wright GA, Kisiolek J, Jones MT, Oliver JM. Position specific changes in body composition, hydration status, and metabolism during preseason training camp and nutritional habits of Division III football players. The Open Sport Sciences Journal. 2016;10:3.

9. Logue, D., et al., Low energy availability in athletes: a review of prevalence, Dietary Patterns, Physiological Health, and Sports Performance. Sports Medicine, 2017.

10. Mountjoy $\mathrm{M}$, et al. $1 \mathrm{OC}$ consensus statement on relative energy deficiency in sport (RED-S): 2018 update. Br J Sports Med. 2018;52(11):687-97.

11. Heaney S, O'Connor $\mathrm{H}$, Naughton G, Gifford J. Towards an understanding of the barriers to good nutrition for elite athletes. Int I Sports Sci Coach. 2008; 3(3):391-401.

12. Heaney S, et al. Nutrition knowledge in athletes: a systematic review. Int J Sport Nutr Exerc Metab. 2011;21(3):248-61.

13. Jacobson BH, Sobonya C, Ransone J. Nutrition practices and knowledge of college varsity athletes: a follow-up. J Strength Cond Res. 2001;15(1):63-8.

14. Rash C, Malinauskas BM, Duffrin MW, Barber-Heidal K, Overton RF. Nutritionrelated knowledge, attitude, and dietary intake of colelge track athletes. Sport J. 2008;11(1):48-55.

15. Rosenbloom CA, Jonnalagadda SS, Skinner R. Nutrition knowledge of collegiate athletes in a division I National Collegiate Athletic Association institution. J Am Diet Assoc. 2002:102(3):418-20.

16. Torres-McGehee TM, et al. Sports nutrition knowledge among collegiate athletes, coaches, athletic trainers, and strength and conditioning specialists. J Athl Train. 2012;47(2):205-11.

17. Nana A, et al. Importance of standardized DXA protocol for assessing physique changes in athletes. Int I Sport Nutr Exerc Metab. 2014.
18. Schofield WN. Predicting basal metabolic rate, new standards and review of previous work. Hum Nutr Clin Nutr. 1985;39(Suppl 1):5-41.

19. Assah FK, et al. Accuracy and validity of a combined heart rate and motion sensor for the measurement of free-living physical activity energy expenditure in adults in Cameroon. Int J Epidemiol. 2011;40(1):112-20.

20. Brage $S$, et al. Reliability and validity of the combined heart rate and movement sensor Actiheart. Eur J Clin Nutr. 2005:59(4):561-70.

21. Zabriskie HA, et al. Energy Status and Body Composition Across a Collegiate Women's Lacrosse Season. Nutrients. 2019:11:2.

22. Hinton PS, et al. Nutrient intakes and dietary behaviors of male and female collegiate athletes. Int J Sport Nutr Exerc Metab. 2004:14(4):389-405.

23. Valliant MW, et al. Nutrition education by a registered dietitian improves dietary intake and nutrition knowledge of a NCAA female volleyball team. Nutrients. 2012;4(6):506-16.

24. Beals KA. Eating behaviors, nutritional status, and menstrual function in elite female adolescent volleyball players. J Am Diet Assoc. 2002;102(9):1293-6.

25. Hoogenboom BJ, et al. Nutritional knowledge and eating behaviors of female, collegiate swimmers. N Am J Sports Phys Ther. 2009;4(3):139-48.

26. Burke $L M$, et al. Guidelines for daily carbohydrate intake: do athletes achieve them? Sports Med. 2001:31(4):267-99.

27. Rossi FE, et al. The effects of a sports nutrition education intervention on nutritional Status, sport nutrition knowledge, body composition, and performance during off season training in NCAA division I baseball players. J Sports Sci Med. 2017;16(1):60-8.

28. Abood DA, Black DR, Birnbaum RD. Nutrition education intervention for college female athletes. J Nutr Educ Behav. 2004:36(3):135-7.

29. Hull MV, et al. Gender differences and access to a sports dietitian influence dietary habits of collegiate athletes. J Int Soc Sports Nutr. 2016;13:38.

30. Hull MV, et al. Availability of a sports dietitian may lead to improved performance and recovery of NCAA division I baseball athletes. J Int Soc Sports Nutr. 2017;14:29.

31. Capling L, et al. Validity of Dietary Assessment in Athletes: A Systematic Review. Nutrients. 2017:9:12.

32. Enemark-Miller EA, Seegmiller JG, Rana SR. Physiological profile of women's lacrosse players. J Strength Cond Res. 2009:23(1):39-43.

33. Maughan RJ, Shirreffs SM. Nutrition and hydration concerns of the female football player. Br J Sports Med. 2007:41(Suppl 1):160-3.

\section{Publisher's Note}

Springer Nature remains neutral with regard to jurisdictional claims in published maps and institutional affiliations.

Ready to submit your research? Choose BMC and benefit from:

- fast, convenient online submission

- thorough peer review by experienced researchers in your field

- rapid publication on acceptance

- support for research data, including large and complex data types

- gold Open Access which fosters wider collaboration and increased citations

- maximum visibility for your research: over $100 \mathrm{M}$ website views per year

At $\mathrm{BMC}$, research is always in progress.

Learn more biomedcentral.com/submissions 